\title{
STUDIES OF QED AND NUCLEAR SIZE EFFECTS WITH HIGHLY CHARGED IONS IN AN EBIT
}

\author{
J. R. CRESPO LÓPEZ-URRUTIA, J. BRAUN, G. BRENNER, H. BRUHNS, \\ A. J. GONZÁLEZ MARTÍNEZ, A. LAPIERRE, V. MIRONOV, R. SORIA \\ ORTS, H. TAWARA, M. TRINCZEK, AND J. ULLRICH \\ Max-Planck-Institute for Nuclear Physics \\ Saupfercheckweg 1 \\ D-69117 Heidelberg, Germany \\ E-mail: crespojr@mpi-hd.mpg.de \\ A. ARTEMYEV ${ }^{\dagger}$, J. H. SCOFIELD ${ }^{\ddagger}$, I. I. TUPITSYN ${ }^{\dagger}$ \\ $\dagger$ St. Petersburg State University, St. Petersburg 198504, Russia \\ ${ }^{\ddagger}$ Lawrence Livermore National Laboratory, Livermore, CA 94550, USA
}

\begin{abstract}
Highly charged ions can be conveniently produced and stored at the Heidelberg electron beam ion trap. Various experiments have been undertaken to exploit the strong scaling of QED effects and nuclear size contributions with the ion charge. Instrumental developments in our group have led to an increased spectroscopic precision. Wavelengths of forbidden transitions in the visible have been measured with sub-ppm accuracy, and their Zeeman splitting has been resolved, thus allowing a direct determination of $\mathrm{g}$-factors, and even the measurement of isotopic recoil effects with relativistic bound electrons. Experiments on the lifetime of metastable levels has yielded results sensitive to hitherto unmeasurable QED contributions of the order of $0.5 \%$, such as that arising from the electron anomalous magnetic moment. In the mid- $\mathrm{Z}$ region, measurements of the Lyman- $\alpha_{1}(\mathrm{H}-$ like $\mathrm{Cl})$ and $1 \mathrm{~s} 2 \mathrm{p}^{1} \mathrm{P}_{1} \rightarrow 1 \mathrm{~s}^{2}{ }^{1} \mathrm{~S}_{0}$ transitions (He-like Ar) have been performed with an accuracy better than $10 \mathrm{ppm}$. Finally, in the dielectronic resonances of very heavy ions, such as $\mathrm{Hg}^{78+\ldots 75+}$, where QED contributions are in the order of $160 \mathrm{eV}$, excitation energies of several tens of $\mathrm{keV}$ have been determined within a few $\mathrm{eV}$ absolute uncertainties, and quantum interference effects studied with state selectivity, allowing a detailed comparison with different predictions.
\end{abstract}

\section{Precision studies with electron beam ion traps}

An electron beam ion trap (EBIT) is an ideal device for precision spectroscopy of highly charged ions (HCI), as the experience from several groups has shown in recent years. The EBIT at the Max-Planck-Institute for Nuclear Physics in Heidelberg ${ }^{1}$ produces and confines HCI by means of a mo- 
noenergetic electron beam compressed by an intense magnetic field. The ions are prepared in well defined charge states, trapped in a small volume under UHV conditions, and their electronic excitation can be tuned, modified and switched on an off easily. Spectroscopic access to the trapped ions enables high resolution measurements and lifetime studies; extracted ions can be used for collision physics studies. Here we present recent results obtained with this device, showing how newer developments have allowed us to become very sensitive to QED contributions and nuclear size effects in a wide range of energies.

\section{Observation of isotopic shifts in Zeeman-splitted forbidden transitions in Ar XIV and Ar XV}

Magnetic dipole (M1) transitions in highly charged ions (HCI) are of great interest to obtain essential information on astrophysical and tokamak plasmas ${ }^{2}$. However, various spectroscopic techniques used so far in experimental studies of such plasmas could not provide a direct observation of the Zeeman splitting because of excessive line broadening caused by the thermal motion of the ions. By a judicious choice of the operation parameters, namely low electron current (20-50 mA), low axial trapping voltage (nominally $0-10 \mathrm{~V})$ and strong magnetic field $(6.82 \mathrm{~T})$ the ion temperature, and thus the Doppler width of the emitted lines was significantly reduced. This made a direct observation of the Zeeman splitting in B-like $\left(\mathrm{Ar}^{13+}\right)$ and Be-like $\left(\mathrm{Ar}^{14+}\right)$ argon ions with an accuracy of better than 0.5 ppm at $441 \mathrm{~nm}$ possible. For the $2 \mathrm{~s}^{2} 2 \mathrm{p}^{2} \mathrm{P}_{1 / 2^{-}}{ }^{2} \mathrm{P}_{3 / 2} \mathrm{Ar}^{13+} \mathrm{M} 1$ transition, six Zeeman components were resolved (see Figure 1). Their polarization was also investigated. For M1 transitions, the central components $(\Delta \mathrm{M}=0)$ are polarized perpendicularly to the field, hence the $\Delta \mathrm{M}=0$ transitions are $\sigma$-components and the $\Delta \mathrm{M}= \pm 1$ are $\pi$-components, in contrast to the case of the E1 transitions. Tens of individually calibrated spectra were acquired with a grating spectrometer, achieving excellent statistics. We have remeasured the central wavelength, obtaining $\lambda=441.2558(1) \mathrm{nm}$, in good agreement with previous measurements ${ }^{3}$. Through the measured line splitting we determined the $g_{J}$ of the upper $(\mathrm{J}=3 / 2)$ and lower $(\mathrm{J}=1 / 2)$ level of the $2 \mathrm{~s}^{2} 2 \mathrm{p}$ state as well. The results of $g_{3 / 2}=1.331(1)$ and $g_{1 / 2}=0.655(3)$ can be compared with theoretical predictions of 1.331665 and 0.664492 , respectively.

Isotopic shifts could also be measured with this setup; the results for the wavelength and the isotopic shifts are compared in Table 1 with cal- 

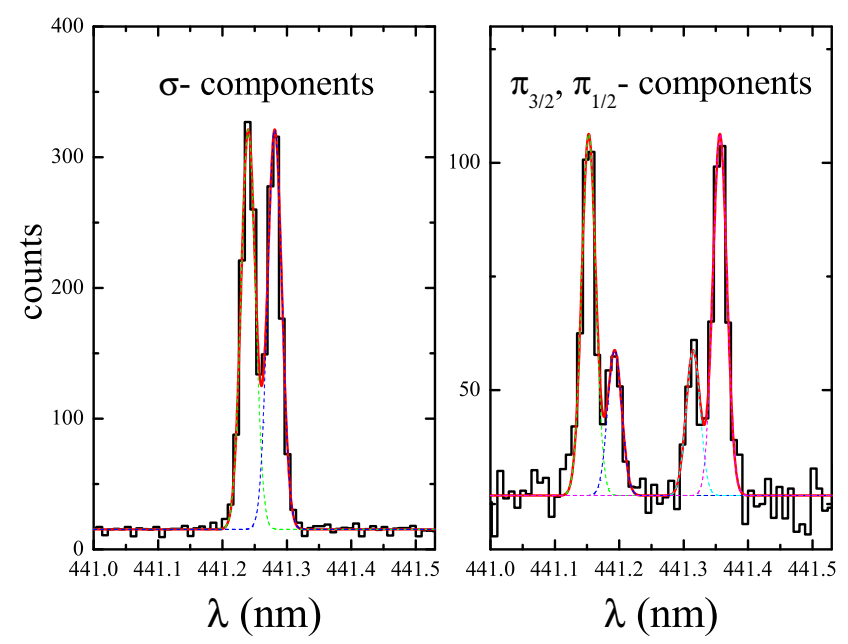

Figure 1. Single spectrum of the M1 line viewed perpendicularly to the magnetic field. (a) $\Delta \mathrm{M}=0$, (b) $\Delta \mathrm{M}= \pm 1$.

Table 1. Wavelengths of the $\mathrm{Ar}^{13+, 14+} \mathrm{M} 1$ lines and their isotope shifts $\left({ }^{40} \mathrm{Ar} /{ }^{36} \mathrm{Ar}\right)$ (in $\left.\mathrm{nm}\right)$.

\begin{tabular}{lcccc}
\hline & \multicolumn{2}{c}{ Wavelength } & \multicolumn{2}{c}{ Isotope shifts } \\
\hline Ion & Theory & Experiment & Theory & Experiment \\
\hline $\boldsymbol{A r}^{13+}$ & $441.16(27)$ & $441.2556(2)$ & 0.00126 & $0.00125(7)$ \\
$\boldsymbol{A r}^{14+}$ & $594.24(30)$ & $594.3879(2)$ & 0.00136 & $0.0012(1)$ \\
\hline
\end{tabular}

culations including second-order Coulomb interaction and Breit-Coulomb interaction, frequency-dependent Breit interaction in lowest order as well as the Lamb shift. A large-scale configuration interaction Dirac-Fock (CIDF) method was used. Here, the largest theoretical uncertainties, which are mainly due to the treatment of electronic correlation and QED corrections, disappear when comparing the two isotopes, revealing the small contribution of the mass shift. In this way, it was possible to observe how the relativistic dynamics of relativistic bound electrons induces isotopic shifts, which up to very recently could not even be predicted properly due to difficult treatment of many-electron relativistic recoil effects. 


\section{High accuracy lifetime measurement of the Ar XIV $1 \mathrm{~s}^{2} 2 \mathrm{~s}^{2} 2 \mathrm{p}^{2} \mathrm{P}_{3 / 2}^{\circ}$ metastable level}

Accurate measurements of atomic transition energies and excited-state lifetimes, besides being important for the diagnostics of tenuous plasmas, are of interest due to their sensitivity to quantum electro-dynamic (QED) effects. Lifetimes reveal non-averaged information on the magnitude of the expansion coefficients in an individual basis of the atomic wavefunction and, hence investigate a widely unexplored facet of current atomic theories. Unfortunately, accurate lifetime measurements still remain an serious experimental challenge due to the difficulty of reaching a high statistical significance allowing to understand, control and model the time evolution of the ion (or atom) population under observation. The Heidelberg EBIT offers the possibility to significantly circumvent these problems thanks to its trapping efficiency, large trap volume and light collection solid angle.

The lifetime of the metastable $1 \mathrm{~s}^{2} 2 \mathrm{~s}^{2} 2 \mathrm{p}^{2} \mathrm{P}_{3 / 2}^{\circ}$ level of boronlike Ar XIV was measured ${ }^{4}$ by monitoring its temporal decay to its ${ }^{2} \mathrm{P}_{1 / 2}^{\circ}$ ground state through the aforementioned M1 transition at $441 \mathrm{~nm}$. The decay was followed for a duration up to 100 times longer than the lifetime to understand and exclude possible systematics effects. Achieving a $0.1 \%$ accuracy level, this result of 9.573(4)(5) ms (stat)(syst) is 17 times more precise than a previous one $9.70(15) \mathrm{ms}^{5}$ and the most accurate ever performed in multiply charged ions. The experimental accuracy had been, up to now, always insufficient for a verification of the theoretical treatments of relativistic correlation and QED effects in high-precision atomic theories. As seen in Figure 2, the result shows a discrepancy of about $3 \sigma$ with the most recent theoretical predictions corrected by the accurate experimental ${ }^{2} \mathrm{P}_{1 / 2}^{\circ}{ }^{-2} \mathrm{P}_{3 / 2}^{\circ}$ transition wavelength and the previously (in almost all cases) neglected contribution of the free electron anomalous magnetic moment (EAMM).

\section{High precision x-ray spectroscopy of hydrogenic and helium-like argon ions}

In order to overcome many of the current difficulties found in the absolute calibration of $\mathrm{x}$-ray spectrometers, a novel method not using any collimation has been implemented in the HD-EBIT flat crystal $x$-ray spectrometer ${ }^{6},{ }^{7}$ making it more suitable for experiments at low $\mathrm{x}$-ray fluxes and eliminating numerous sources of systematic error. The central point of the technique is to measure and monitor the exact position on the crystal where the monoenergetic $\mathrm{x}$-rays are reflected in dependence of the mea- 


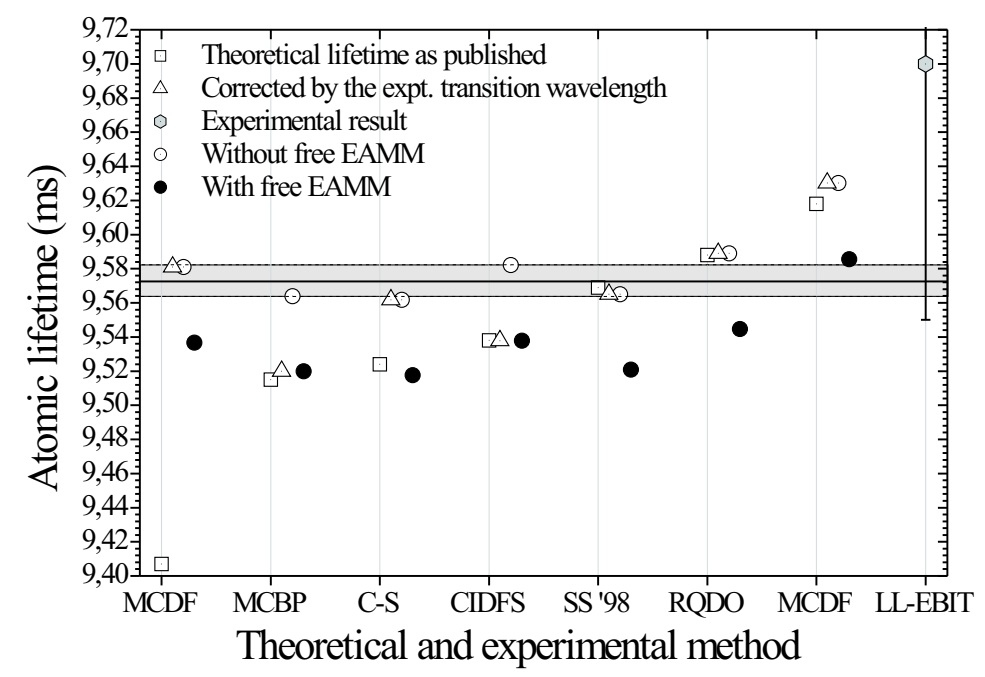

Figure 2. Theoretical lifetimes with and without EAMM corrections compared with experimental results.

sured crystal angle instead of defining it through collimation. Two light beams projected onto the Bragg crystal are used as fiducials for this purpose. Unlike the $\mathrm{x}$-rays, which have to fulfill Bragg's law, light is reflected by the crystal surface under any angle, and a rotation results in a shift of the $\mathrm{x}$-ray reflection position with respect to the fiducials' reflection positions on the crystal. Consequently, the position at which the CCD camera detects the x-rays moves in relation to the light fiducials. The three relative positions yield full information about the reflection position of the $\mathrm{x}$-ray line on the crystal and therefore very accurate Bragg angle measurements.

By using this technique, the transition $1 \mathrm{~s} 2 \mathrm{p}^{1} \mathrm{P}_{1} \rightarrow 1 \mathrm{~s}^{2}{ }^{1} \mathrm{~S}_{0}$ of He-like argon $\mathrm{Ar}^{16+}$ (commonly referred to as $w$-line) has been measured at the HD-EBIT with respect to the Lyman- $\alpha_{1}$ transition of H-like argon, which is known to a precision of $5 \mathrm{ppm}^{8}$. About 50 x-ray exposures at the expected Bragg angles (with small deviations to actively change the reflection position on the crystal) of the Lyman- $\alpha_{1}$ and $w$ transition have been acquired, all of them preceded and followed by a visible light exposure. For each of these sets of spectra the relative position of the x-ray line to the fiducials was obtained and plotted over the corresponding measured crystal angle. From this plot the difference of Bragg angles of the two lines was extracted. With this angular difference and the literature value of the Lyman- $\alpha_{1}$ transition energy, the result for the $w$ transition energy 
is $3139.538(21) \mathrm{eV}$, in good agreement with the best available experimental result of $3139.552(37) \mathrm{eV}^{9}$. In this way, a precision of $7 \mathrm{ppm}$ for the $w$ and $z$ transitions in helium-like argon, and of 9 ppm for the Lyman- $\alpha_{1}$ transition in $\mathrm{H}$-like $\mathrm{Cl}^{16+}$ was reached using the $\mathrm{Ar}^{17+}$ Lyman- $\alpha_{1}$ transition as reference. This result is in excellent agreement with the theoretical prediction. The Dirac energy for the $\mathrm{Cl}^{16+}$ Lyman- $\alpha_{1}$ transition is $2963.310 \mathrm{eV}$. The total Lamb shift of $-0.947 \mathrm{eV}^{10}$ includes a contribution from the vacuum polarisation of $0.068 \mathrm{eV}$. Since the uncertainties of previous measurements of this transition were $100 \mathrm{meV}$ or larger, the present measurement is the first one sensitive to resolving the vacuum polarisation contribution in $\mathrm{H}$ like $\mathrm{Cl}^{16+}$ and achieves a sensitivity to the total Lamb shift on a level of better than $3 \%$. The precision reached in the $\mathrm{Ar}^{16+} w$ transition energy measurement corresponds to $1.9 \%$ of the one-electron QED contribution to the ground state binding energy, and it even probes the two-electron QED contribution at a level of $22 \%$. In the case of the $z$ line, the one-electron QED contribution to the excited ${ }^{3} S_{1}$ state can also be resolved.

Currently, the setup is being modified in order to perform absolute wavelength measurements and completely eliminate the need for reference lines, aiming at a precision approaching 1 ppm or better.

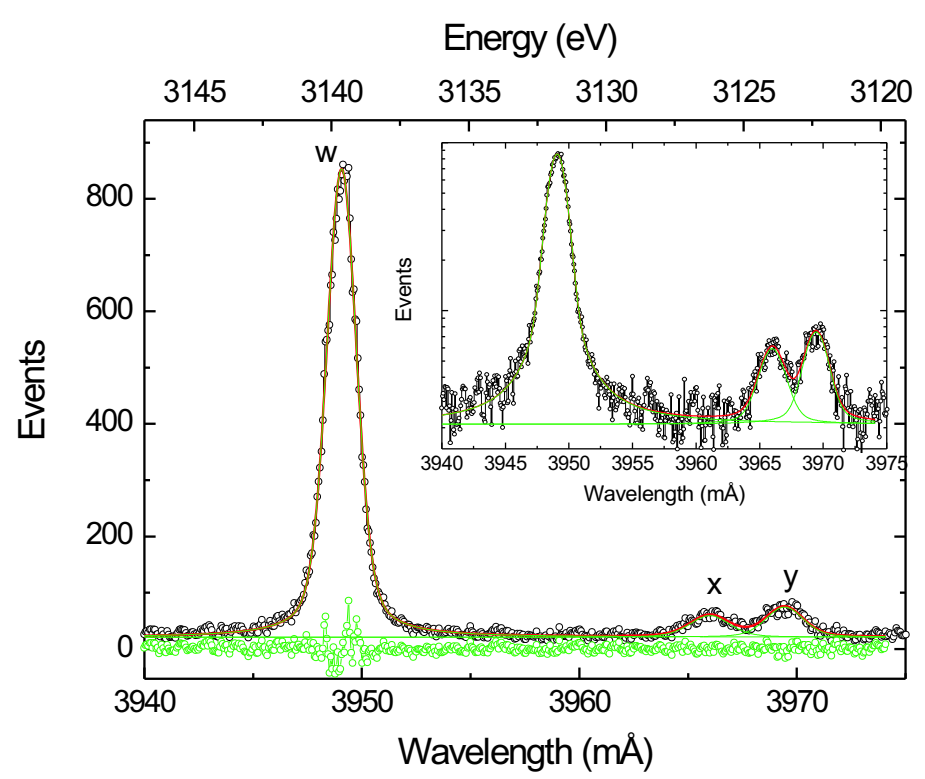

Figure 3. The $\mathrm{Ar}^{16+} w$ transition at $3140 \mathrm{eV}$. 


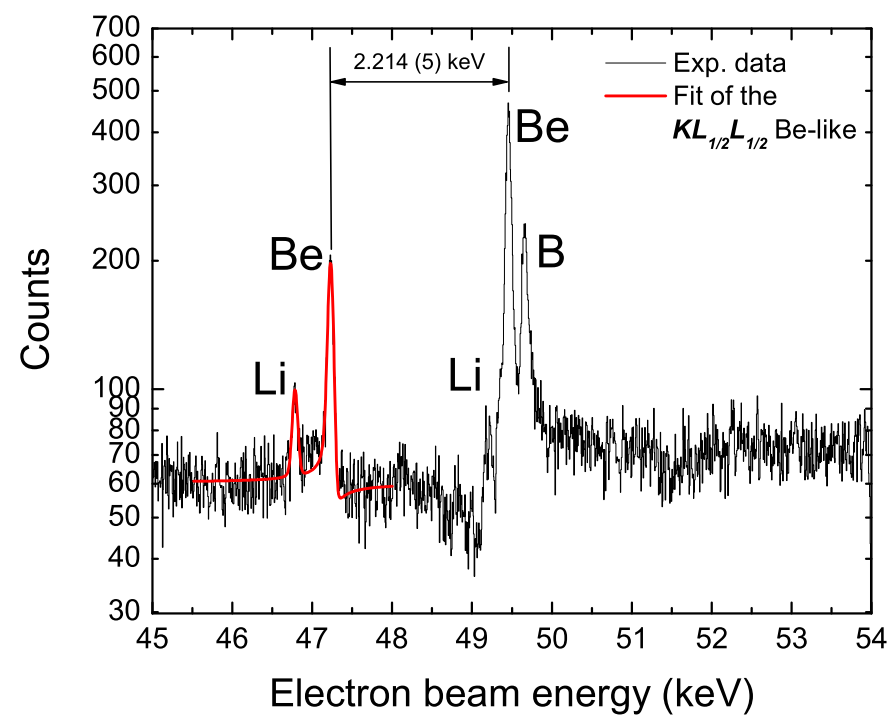

Figure 4. Projection of the Be-like $\mathrm{DR}$ resonances on the in the $\mathrm{KL}_{1 / 2} \mathrm{~L}_{1 / 2}$ (left) and $\mathrm{KL}_{1 / 2} \mathrm{~L}_{3 / 2}$ (right) region. Small contributions of Li-like and B-like resonances can be seen at higher electron energies.

\section{Quantum interference and fine structure splitting in the photorecombination of $\left(\mathrm{Hg}^{78+\ldots 75+}\right)$ ions}

Heavy HCI allow one to test in detail QED contributions in strong fields, as well as finite nuclear size effects, since these effects scale tremendously with high powers of the ions' nuclear charge $Z$; e.g., the calculated QED contributions to the energy of the 1s electron in mercury ions are about 160 $\mathrm{eV}$, and nuclear size effects are of the order of $50 \mathrm{eV}$. In this experiment, while scanning the electron beam energy of the EBIT, the energies of the photons emitted due to photorecombination are recorded event by event and displayed versus the beam energy in two-dimensional plots. Distinct structures in radiative recombination $(\mathrm{RR})$ and dielectronic recombination (DR) processes appear in the plots. Here, the excitation energies of the KLL $\mathrm{DR}$ resonances of mercury ions ranging from He-like $\mathrm{Hg}^{78+}$ to B-like $\mathrm{Hg}^{75+}$ are determined within a few $\mathrm{eV}$ at $50 \mathrm{keV}$ using a temperature-stabilized high precision voltage divider. The fine structure splitting between states with different total angular momentum $J$ are even more accurately extracted by measuring voltage differences. The splitting between the states $\left[\left(1 s 2 s^{2} 2 p_{1 / 2}\right)_{0} 2 p_{3 / 2}\right]_{3 / 2}$ and $\left[1 s 2 s^{2}\left(2 p_{1 / 2}\right)^{2}\right]_{1 / 2}$ of Be-like ions in the initial 
state was determined and compared with different calculations ${ }^{1211}$. Small, yet significant discrepancies were found for the absolute excitation energies of the Li-, Be- and B-like ions, while the He-like results showed excellent agreement with theory. Additionally, by projecting narrow slices of the RR bands containing photon events arising mostly from a single charge state onto the electron beam energy axis (see Figure 4) we are able to study the photorecombiantion process for well-resolved electronic states of that individual charge state. The observed resonance curves were fitted with asymmetric Fano line profiles caused by the interference between DR and $\mathrm{RR}$ recombination channels ${ }^{13}$, yielding state-resolved Fano-factors.

\section{References}

1. J. R. Crespo López-Urrutia, A. Dorn, R. Moshammer, and J. Ullrich, Phys. Scr. T80, 502 (1999)

2. U. Feldman, J. F. Seely, N. R. Sheeley, S. Suckewer, and A. M. Title, J. Appl. Phys. 56, 2341 (1984)

3. I. N. Draganić, J. R. Crespo López-Urrutia, R. Dubois, S. Fritsche, V. M. Shabaev, R. Soria Orts, I. I. Tupitsyn, Y. Zou, and J. Ullrich, Phys. Rev. Lett. 91, 183001 (2003)

4. A. Lapierre, U. D. Jentschura, J. R. Crespo López-Urrutia, J. Braun, G. Brenner, H. Bruhns, D. Fischer, A. J. González Martínez, Z. Harman, W. R. Johnson, C. H. Keitel, V. Mironov, C. J. Osborne, G. Sikler, R. Soria Orts, H. Tawara, I. I. Tupitsyn, J. Ullrich, and A. Volotka, accepted in Phys. Rev. Lett. (2005)

5. E. Träbert, P. Beiersdorfer, S. B. Utter, G. V. Brown, H. Chen, C. L. Harris, P. A. Neill, D. W. Savin, and A. J. Smith, Astrophys. J. 541, 506 (2000)

6. J. Braun, Diploma thesis, 2003, MPI-K Heidelberg

7. J. Braun, H. Bruhns, M. Trinczek, J. R. Crespo López-Urrutia, and J. Ullrich, Rev. Sci. Instrum.76, 073105 (2005)

8. H. F. Beyer, R. D. Deslattes, F. Folkmann, R. E. LaVilla, J. Phys. B: At. Mol. Phys. 18, 207 (1985)

9. R. D. Deslattes, H. F. Beyer, F. Folkmann, J. Phys. B: At. Mol. Phys. 17, L689-L694 (1984)

10. A. N. Artemyev, V. M. Shabaev, V. A. Yerokhin, G. Plunien and G. Soff, Phys. Rev. A 71, 062104 (2005)

11. J. H. Scofield, Phys. Rev. A 40, 3054 (1989)

12. A. Artemyev, V. M. Shabaev, M. M. Sysak, V. A. Yerokhin, T. Beier, G. Plunien and G. Soff, Phys. Rev. A 67, 062506 (2003)

13. A. J. González Martínez, J. R. Crespo López-Urrutia, J. Braun, G. Brenner, H. Bruhns, A. Lapierre, V. Mironov, R. Soria Orts, H. Tawara, M. Trinczek, and J. Ullrich, Phys. Rev. Lett. 94, 203201 (2005) 